\title{
Linear optical absorption spectra of mesoscopic structures in intense THz fields: Free- particle properties
}

\author{
Johnsen, Kristinn; Jauho, Antti-Pekka
}

Published in:

Physical Review B

Link to article, DOI:

10.1103/PhysRevB.57.8860

Publication date:

1998

Document Version

Publisher's PDF, also known as Version of record

Link back to DTU Orbit

Citation (APA):

Johnsen, K., \& Jauho, A-P. (1998). Linear optical absorption spectra of mesoscopic structures in intense $\mathrm{THz}$ fields: Free-particle properties. Physical Review B, 57(15), 8860-8872.

https://doi.org/10.1103/PhysRevB.57.8860

\section{General rights}

Copyright and moral rights for the publications made accessible in the public portal are retained by the authors and/or other copyright owners and it is a condition of accessing publications that users recognise and abide by the legal requirements associated with these rights.

- Users may download and print one copy of any publication from the public portal for the purpose of private study or research.

- You may not further distribute the material or use it for any profit-making activity or commercial gain

- You may freely distribute the URL identifying the publication in the public portal

If you believe that this document breaches copyright please contact us providing details, and we will remove access to the work immediately and investigate your claim. 


\title{
Linear optical absorption spectra of mesoscopic structures in intense THz fields: Free-particle properties
}

\author{
Kristinn Johnsen \\ Mikroelektronik Centret, Technical University of Denmark, Building 345east, DK-2800 Lyngby, Denmark \\ and Department of Mathematics, University of California, Santa Barbara, California 93106 \\ Antti-Pekka Jauho \\ Mikroelektronik Centret, Technical University of Denmark, Building 345east, DK-2800 Lyngby, Denmark
}

(Received 3 April 1997; revised manuscript received 26 November 1997)

\begin{abstract}
We theoretically study the effect of THz radiation on the linear optical absorption spectra of semiconductor structures. A general theoretical framework, based on nonequilibrium Green functions, is formulated and applied to the calculation of linear optical absorption spectrum for several nonequilibrium mesoscopic structures. We show that a blueshift occurs and sidebands appear in bulklike structures, i.e., the dynamical FranzKeldysh effect [A.-P. Jauho and K. Johnsen, Phys. Rev. Lett. 76, 4576 (1996)]. An analytic calculation leads to the prediction that in the case of superlattices distinct stable steps appear in the absorption spectrum when conditions for dynamical localization are met. [S0163-1829(98)03412-2]
\end{abstract}

\section{INTRODUCTION}

Light absorption can be described in terms of a process where polarization is induced into the medium. To linear order in the electric-field component of the traversing light, $\mathcal{E}$, the induced polarizability $\mathcal{P}$ can be expressed in terms of the dielectric susceptibility $\chi$ as

$$
\mathcal{P}(t)=\int_{-\infty}^{t} d t^{\prime} \chi\left(t, t^{\prime}\right) \mathcal{E}\left(t^{\prime}\right)
$$

If the absorbing medium is in a stationary state, the susceptibility depends only on the difference of its time arguments, i.e., $\chi\left(t, t^{\prime}\right)=\chi\left(t-t^{\prime}\right)$. Under these conditions Maxwell's equation for $\mathcal{E}$ is an algebraic equation in frequency space, and one finds that the absorption is proportional to the imaginary part of $\chi(\omega)$. However, under nonequilibrium conditions, which are the topic of the present study, the susceptibility is a two-time function, and Maxwell's equation remains an integral equation even in the frequency domain. Further progress hinges upon two steps: first, one has to develop methods to calculate the nonequilibrium susceptibility function, and, second, one has to specify what sort of lightwave or pulse is used in the absorption experiment. The present work addresses both of these problems. As far as the time dependence of the probe pulse is concerned, two specific situations are examined. First, consider an undoped semiconductor placed in an intense $\mathrm{THz}$ field; we assume that the THz field is not able to induce polarization, i.e., no carriers are excited in the conduction band. Such a system is in a nonequilibrium state, i.e., the susceptibility is a two-time function. Properties of such systems were investigated experimentally using the free-electron laser (FEL) as a source for intense $\mathrm{THz}$ fields, ${ }^{1}$ many interesting properties were discovered, and others predicted, such as photon-assisted tunneling, ${ }^{2}$ dynamical localization and absolute negative conductivity, ${ }^{3}$ the ac Stark effect, ${ }^{4}$ the dynamical FranzKeldysh effect, and the formation of sidebands. ${ }^{5-9}$ A second example consists of ultrafast transients. Consider an undoped semiconductor structure subject to an external intense static field. At some time instant a population of carriers is pumped into the conduction band. These mobile charges will rearrange themselves so as to screen the external field. While the screening is building up, the susceptibility of the system is a two-time function. Using femtosecond laser techniques, which are able to probe the time scales in which screening is building up, experiments investigating the nonequilibrium properties of such systems have been performed. ${ }^{10,11}$

Band-gap engineering techniques of semiconductor compounds, such as molecular-beam epitaxy, allow spatial modulation of the band gap down to atomic resolution. It is possible to break the translational symmetries of bulk crystals, induce new ones, and reduce the degrees of freedom with these techniques. Construction of systems which are effectively two dimensional (2D) and even one dimensional (1D) with regard to electron mobility and optical properties is today a standard procedure. Another example of such manmade structures are superlattices (SL's), i.e., an engineered periodic potential in the growth direction of the sample. The interplay between the mesoscopic properties and the dynamical properties can lead to many interesting phenomena such as absolute negative resistance for the transport properties, ${ }^{12}$ and the rich features in the optical properties which are the subject of the present work.

The purpose of this paper is to present a theoretical study of light absorption in mesoscopic systems subject to intense $\mathrm{THz}$ [far-infrared (FIR)] fields. We consider undoped systems, which implies that there are no carriers in the conduction band. Thus near-infrared (NIR) interband absorption is the dominant absorption process. The nonequilibrium nature of the system necessitates the use of special theoretical tools; we have chosen to apply nonequilibrium Green-function techniques. ${ }^{13,14}$ In particular, this method allows us to treat the intense FIR field nonperturbatively, and defines a framework in which screening can be treated systematically. Our analysis consists of the following steps. Starting from a two- 
band Hamiltonian, we derive a formal expression for the interband susceptibility in terms of nonequilibrium Green functions. Next, we use the general expression to derive the NIR absorption spectrum for noninteracting particles (Coulomb interactions will be discussed in a subsequent paper; see also below). Finally, we give explicit results for a number of special cases $(3 \mathrm{D}, 2 \mathrm{D}$, and $1 \mathrm{D}$ systems and superlattices) and discuss the physical implications. The relation of our work to previous papers addressing similar topics can be summarized as follows. Bulk systems were studied previously in Refs. 5 and 6, where the relevant energy scales were recognized, and the blueshift of the band edge (see below) was predicted. The photonic subband structure, which is an essential feature of the present work, was not resolved, however. Concerning absorption in superlattices, in a recent work $^{15}$ a detailed numerical study, using Monte Carlo methods, was presented. In the present work we are, by analytic means, able to explain the "surprising" emergence of satellite peaks in the spectrum which escaped explanation on basis of the numerical work.

This paper is organized as follows. In Sec. II we derive a general expression for the two-time dielectric interband susceptibility. Section III relates the susceptibility to the measured absorption, considering both continuous-wave measurements as well as short white light pulses. The singleparticle Green functions and the corresponding spectral functions, which determine the susceptibility, are defined in Sec. IV, and related to the generalized density of states ${ }^{7}$ (GDOS), which, in turn, is shown to determine the optical absorption. Section V considers bulklike systems, and we obtain analytic results for the GDOS, which is analyzed in some detail in terms of the sideband picture. The properties of light absorption in superlattices are treated in Sec. VI. Specific attention is paid to conditions where dynamical localization occurs, and we show how it affects the absorption spectrum. Finally, in Sec. VII we make some concluding remarks.

\section{DIELECTRIC INTERBAND SUSCEPTIBILITY}

We shall now derive an expression for the dielectric interband susceptibility using nonequilibrium Green functions. The microscopic operator describing interband polarization is

$$
\vec{P}(t)=\sum_{k} \vec{d}_{k}\left[a_{k}^{\dagger}(t) b_{k}(t)+b_{k}^{\dagger}(t) a_{k}(t)\right]
$$

Here $\vec{d}_{k}$ is the dipole matrix element, $a_{k}^{\dagger}(t)\left[a_{k}(t)\right]$ are the conduction-band electron creation (annihilation) operators, and $b_{k}^{\dagger}(t)\left[b_{k}(t)\right]$ are the valence-band creation (annihilation) operators. The linearized Hamiltonian associated with a polarization $\vec{P}(t)$ induced by the external field $\mathcal{E}(t)$ is $H_{P}(t)=-\vec{P}(t) \cdot \mathcal{E}(t)$. Linear-response theory now yields the Cartesian $l$ component of the induced interband polarization due to a weak external field $\mathcal{E}$ :

$$
\mathcal{P}_{l}(t)=-\frac{i}{\hbar} \int_{-\infty}^{\infty} d t^{\prime} \theta\left(t-t^{\prime}\right)\left\langle\left[\vec{P}\left(t^{\prime}\right), P_{l}(t)\right]\right\rangle \cdot \mathcal{E}\left(t^{\prime}\right)
$$

The retarded susceptibility tensor can be identified from Eq. (2),

$$
\chi_{l m}^{r}\left(t, t^{\prime}\right)=-\frac{i}{\hbar} \theta\left(t-t^{\prime}\right)\left\langle\left[P_{m}\left(t^{\prime}\right), P_{l}(t)\right]\right\rangle .
$$

Following the standard line of attack in nonequilibrium theory, ${ }^{13}$ we first consider the causal (time-ordered) response function

$$
\chi_{l m}^{c}\left(t, t^{\prime}\right)=-\frac{i}{\hbar}\left\langle T\left\{P_{m}\left(t^{\prime}\right) P_{l}(t)\right\}\right\rangle,
$$

where $T$ is the time-ordering operator. In nonequilibrium, the causal response function is replaced by its contour ordered counterpart $\chi_{l m}^{c}\left(t, t^{\prime}\right) \rightarrow \chi_{l m}^{c}\left(\tau, \tau^{\prime}\right)$, where the complex-time variables $\tau$ and $\tau^{\prime}$ reside on the Keldysh contour. Finally we obtain the retarded tensor by an analytic continuation using the Langreth rules. ${ }^{16}$

We use Eq. (1) to write the susceptibility as

$$
\begin{aligned}
\chi_{l m}^{c}\left(\tau, \tau^{\prime}\right)= & -\frac{i}{\hbar} \sum_{q k} d_{l}(k) d_{m}(q)\left[\left\langleT _ { c } \left\{a_{q}^{\dagger}\left(\tau^{\prime}\right) b_{q}\left(\tau^{\prime}\right)\right.\right.\right. \\
& \left.\left.\times a_{k}^{\dagger}(\tau) b_{k}(\tau)\right\}\right\rangle \\
& +\left\langle T_{c}\left\{a_{q}^{\dagger}\left(\tau^{\prime}\right) b_{q}\left(\tau^{\prime}\right) b_{k}^{\dagger}(\tau) a_{k}(\tau)\right\}\right\rangle \\
& +\left\langle T_{c}\left\{b_{q}^{\dagger}\left(\tau^{\prime}\right) a_{q}\left(\tau^{\prime}\right) a_{k}^{\dagger}(\tau) b_{k}(\tau)\right\}\right\rangle \\
& \left.+\left\langle T_{c}\left\{b_{q}^{\dagger}\left(\tau^{\prime}\right) a_{q}\left(\tau^{\prime}\right) b_{k}^{\dagger}(\tau) a_{k}(\tau)\right\}\right\rangle\right],
\end{aligned}
$$

where $T_{c}$ is the contour-ordering operator. In equilibrium, the two-particle correlation functions occurring in Eq. (5) would be found via the Bethe-Salpeter equation. ${ }^{17}$ In what follows, however, we shall consider the noninteracting limit of Eq. (5). This approach is motivated by the following considerations. The noninteracting limit will allow significant analytic progress, and the results, which we believe are interesting in their own right, form the basis for an interacting theory, to be reported elsewhere (see also below). Second, the experimental findings of absorption in quantum wells, subject to intense FIR, ${ }^{18}$ can be largely understood on the basis of the concepts presented here. A quantitative assessment requires a nonequilibrium theory for the two-particle Green functions, i.e., a numerical solution of the nonequilibrium Bethe-Salpeter equation. We have recently completed this program, and give our results in a subsequent paper. For noninteracting particles we can use Wick's theorem to factorize the two-particle correlation functions. Thus the nonequilibrium susceptibility can be expressed in terms of single-particle Green functions. The following Green functions are needed:

$$
\begin{aligned}
& g_{c}\left(k, \tau ; q, \tau^{\prime}\right)=-i\left\langle T_{c}\left\{a_{k}(\tau) a_{q}^{\dagger}\left(\tau^{\prime}\right)\right\}\right\rangle, \\
& g_{v}\left(k, \tau ; q, \tau^{\prime}\right)=-i\left\langle T_{c}\left\{b_{k}(\tau) b_{q}^{\dagger}\left(\tau^{\prime}\right)\right\}\right\rangle, \\
& g_{a b}\left(k, \tau ; q, \tau^{\prime}\right)=-i\left\langle T_{c}\left\{a_{k}(\tau) b_{q}^{\dagger}\left(\tau^{\prime}\right)\right\}\right\rangle,
\end{aligned}
$$

and

$$
g_{b a}\left(k, \tau ; q, \tau^{\prime}\right)=-i\left\langle T_{c}\left\{b_{k}(\tau) a_{q}^{\dagger}\left(\tau^{\prime}\right)\right\}\right\rangle .
$$


We assume that the frequency $\Omega$ of the FIR field is such that $\hbar \Omega \ll \epsilon_{g}$. In typical experiments on III-V systems $\epsilon_{g}$ is of the order of an $\mathrm{eV}$, while $\hbar \Omega$ is a few $\mathrm{meV}$, so this condition is satisfied. Consequently, interband transitions due to the perturbing field can be ignored, and the Green functions related to the Zener effect, i.e., $g_{a b}\left(k, \tau ; q, \tau^{\prime}\right)$ and $g_{b a}\left(k, \tau ; q, \tau^{\prime}\right)$, are neglected from this on. The first-order nonequilibrium susceptibility thus reads

$$
\begin{aligned}
\chi_{l m}^{c}\left(\tau, \tau^{\prime}\right)= & -\frac{i}{\hbar} \sum_{q k} d_{l}(k) d_{m}(q)\left[g_{c}\left(k, \tau ; q, \tau^{\prime}\right) g_{v}\left(q, \tau^{\prime} ; k, \tau\right)\right. \\
& \left.+g_{v}\left(k, \tau ; q, \tau^{\prime}\right) g_{c}\left(q, \tau^{\prime} ; k, \tau\right)\right] .
\end{aligned}
$$

The analytic continuation to real times is performed with the Langreth rules, ${ }^{16}$ which state that if on contour

$$
C\left(\tau, \tau^{\prime}\right)=A\left(\tau, \tau^{\prime}\right) B\left(\tau^{\prime}, \tau\right),
$$

then the retarded function on the real-time axis is

$$
C^{r}\left(t, t^{\prime}\right)=A^{<}\left(t, t^{\prime}\right) B^{a}\left(t^{\prime}, t\right)+A^{r}\left(t, t^{\prime}\right) B^{<}\left(t^{\prime}, t\right) .
$$

We thus have

$$
\begin{aligned}
\chi_{l m}^{r}\left(t, t^{\prime}\right)= & -\frac{i}{\hbar} \sum_{k} d_{l}(k) d_{m}(k)\left[g_{c}^{<}\left(k, t, t^{\prime}\right) g_{v}^{a}\left(k, t^{\prime}, t\right)\right. \\
& +g_{c}^{r}\left(k, t, t^{\prime}\right) g_{v}^{<}\left(k, t^{\prime}, t\right)+g_{v}^{<}\left(k, t, t^{\prime}\right) g_{c}^{a}\left(k, t^{\prime}, t\right) \\
& \left.+g_{v}^{r}\left(k, t, t^{\prime}\right) g_{c}^{<}\left(k, t^{\prime}, t\right)\right],
\end{aligned}
$$

with

$$
\begin{gathered}
g^{<}\left(t, t^{\prime}\right)=i\left\langle c^{\dagger}\left(t^{\prime}\right) c(t)\right\rangle, \\
g^{a}\left(t, t^{\prime}\right)=i \theta\left(t^{\prime}-t\right)\left\langle\left\{c(t), c^{\dagger}\left(t^{\prime}\right)\right\}\right\rangle, \\
g^{r}\left(t, t^{\prime}\right)=-i \theta\left(t-t^{\prime}\right)\left\langle\left\{c(t), c^{\dagger}\left(t^{\prime}\right)\right\}\right\rangle .
\end{gathered}
$$

We recall the following relations:

$$
\begin{gathered}
{\left[g^{<}\left(t, t^{\prime}\right)\right]^{*}=-g^{<}\left(t^{\prime}, t\right),} \\
{\left[g^{a}\left(t, t^{\prime}\right)\right]^{*}=g^{r}\left(t^{\prime}, t\right),} \\
{\left[g^{r}\left(t, t^{\prime}\right)\right]^{*}=g^{a}\left(t^{\prime}, t\right) .}
\end{gathered}
$$

For certain applications, e.g., Sec. III A, it is convenient to introduce the center of mass variables $T=\left(t^{\prime}+t\right) / 2$ and $\tau=t-t^{\prime} .{ }^{19}$ In terms of these variables the symmetry relations of the Green functions are

$$
\begin{gathered}
{\left[g^{<}(T, \tau)\right]^{*}=-g^{<}(T,-\tau),} \\
{\left[g^{a}(T, \tau)\right]^{*}=g^{r}(T,-\tau),} \\
{\left[g^{r}(T, \tau)\right]^{*}=g^{a}(T,-\tau) .}
\end{gathered}
$$

The retarded susceptibility expressed in center-of-mass coordinates is

$$
\begin{aligned}
\chi_{l m}^{r}(T, \tau)= & -\frac{i}{\hbar} \sum_{k} d_{l}(k) d_{m}(k)\left\{\left[g_{c}^{<}(k, T, \tau) g_{v}^{a}(k, T,-\tau)\right.\right. \\
& \left.\left.+g_{v}^{<}(k, T, \tau) g_{c}^{a}(k, T,-\tau)\right]- \text { H.c. }\right\}
\end{aligned}
$$

Note that in equilibrium $\chi_{l m}^{r}(T, \tau)=\chi_{l m}^{r}(\tau)$. As shown in Sec. III A, the relevant quantity for continuous-wave measurements at frequency $\omega_{l}$ is

$$
\operatorname{Im} \chi_{l m}^{r}\left(T, \omega_{l}\right)=\operatorname{Im}\left\{\int_{-\infty}^{\infty} d \tau e^{i \omega_{l} \tau} \chi_{l m}^{r}(T, \tau)\right\}
$$

to first order in $\Omega / \omega_{l}$ (here $\Omega$ is the FIR frequency). Now $\chi_{l m}^{r}(T, \tau)$ is a real quantity, as is evident from Eq. (19). Using the properties of the Fourier transform, we obtain

$$
\begin{aligned}
\chi_{l m}^{r}\left(T, \omega_{l}\right)= & -\frac{i}{\hbar} \sum_{k} d_{l}(k) d_{m}(k) \int_{-\infty}^{\infty} \frac{d \omega}{2 \pi}\left\{g_{c}^{<}(k, T, \omega)\right. \\
& \times\left[g_{v}^{a}\left(k, T, \omega-\omega_{l}\right)+g_{v}^{r}\left(k, T, \omega+\omega_{l}\right)\right] \\
& +g_{v}^{<}(k, T, \omega)\left[g_{c}^{a}\left(k, T, \omega-\omega_{l}\right)\right. \\
& \left.\left.+g_{c}^{r}\left(k, T, \omega+\omega_{l}\right)\right]\right\} .
\end{aligned}
$$

Since $\chi_{l m}^{r}(T, \tau)$ is real, the imaginary part of its Fourier transform is obtained through

$$
\operatorname{Im} \chi_{l m}^{r}\left(T, \omega_{l}\right)=\frac{1}{2 i}\left[\chi_{l m}^{r}\left(T, \omega_{l}\right)-\chi_{l m}^{r}\left(T,-\omega_{l}\right)\right] .
$$

We can therefore write, in terms of the spectral functions

$$
A_{c}(k, T, \omega)=i\left[g_{c}^{r}(k, T, \omega)-g_{c}^{a}(k, T, \omega)\right]
$$

and

$$
A_{v}(k, T, \omega)=i\left[g_{v}^{r}(k, T, \omega)-g_{v}^{a}(k, T, \omega)\right],
$$

that

$$
\begin{aligned}
\operatorname{Im} \chi_{l m}^{r}\left(T, \omega_{l}\right)= & \frac{i}{2 \hbar} \sum_{k} d_{l}(k) d_{m}(k) \int_{-\infty}^{\infty} \frac{d \omega}{2 \pi}\left\{g_{c}^{<}(k, T, \omega)\right. \\
& \times\left[A_{v}\left(k, T, \omega-\omega_{l}\right)-A_{v}\left(k, T, \omega+\omega_{l}\right)\right] \\
& +g_{v}^{<}(k, T, \omega)\left[A_{c}\left(k, T, \omega-\omega_{l}\right)\right. \\
& \left.\left.-A_{c}\left(k, T, \omega+\omega_{l}\right)\right]\right\} .
\end{aligned}
$$

The lesser functions can be expressed in the form ${ }^{13}$

$$
g_{a}^{<}(k, T, \omega)=i f_{a}(k, T, \omega) A_{a}(k, T, \omega),
$$

where $f_{a}(k, T, \omega)$ is a generalized particle distribution for particles of species $a$, and $A_{a}(k, T, \omega)$ is the corresponding spectral function. In accordance with our assumption about no FIR field-induced interband transitions, we can set $f_{c}(k, T, \omega)=0$ (zero occupation of conduction band), and that $f_{v}(k, T, \omega)=1$ (all valence states are occupied). In the general case, e.g., when considering nonlinear effects in the probing light field, one would have to find $f_{a}(k, T, \omega)$ via, say, a Monte Carlo solution of semiconductor Bloch equations $\mathrm{s}^{20,21}$ or by a direct integration of quantum kinetic equations for $g_{a}^{<}(k, T, \omega) .{ }^{13}$ With these assumptions the susceptibility reduces to 


$$
\begin{aligned}
\operatorname{Im} \chi_{l m}^{r}\left(T, \omega_{l}\right)= & \frac{-1}{2 \hbar} \sum_{k} d_{l}(k) d_{m}(k) \int_{-\infty}^{\infty} \frac{d \omega}{2 \pi} A_{v}(k, T, \omega) \\
& \times\left\{A_{c}\left(k, T, \omega-\omega_{l}\right)-A_{c}\left(k, T, \omega+\omega_{l}\right)\right\} \\
= & \frac{1}{2 \hbar} \sum_{k} d_{l}(k) d_{m}(k) \int_{-\infty}^{\infty} \frac{d \omega}{2 \pi} A_{v}(k, T, \omega) \\
& \times A_{c}\left(k, T, \omega+\omega_{l}\right) .
\end{aligned}
$$

The second equality comes about because we do not consider overlapping bands. Equation (27), which is the central result of this section, expresses the fact that the nonequilibrium interband susceptibility function can be calculated from a joint spectral function, which is a convolution of the individual band spectral function. A similar result is known from high-field quantum transport theory: ${ }^{22}$ there the fielddependent scattering rate is expressed as a joint spectral function for the initial and final states.

In order to make a connection to the equilibrium case, we recall the exact identity $g_{\text {eq }}^{<}(k, \omega)=i a_{\text {eq }}(k, \omega) n(\omega)(n(\omega)$ is the Fermi function), and from Eq. (25) (Refs. 17 and 23) we obtain

$$
\begin{aligned}
\operatorname{Im} \chi_{l m}^{r}\left(\omega_{l}\right)= & -\frac{1}{2 \hbar} \sum_{k} d_{l}(k) d_{m}(k) \int_{-\infty}^{\infty} \frac{d \omega}{2 \pi}\left\{n_{c}(\omega) a_{c, \mathrm{eq}}(k, \omega)\left[a_{v, \mathrm{eq}}\left(k, \omega-\omega_{l}\right)-a_{v, \mathrm{eq}}\left(k, \omega+\omega_{l}\right)\right]+n_{v}(\omega) a_{v, \mathrm{eq}}(k, \omega)\right. \\
& \left.\times\left[a_{c, \mathrm{eq}}\left(k, \omega-\omega_{l}\right)-a_{c, \mathrm{eq}}\left(k, \omega+\omega_{l}\right)\right]\right\} .
\end{aligned}
$$

Here $n_{c}(\omega)$ is the conduction-band electron occupation function, and $n_{v}(\omega)$ is the corresponding function for the valence-band electrons.

\section{ABSORPTION COEFFICIENT IN TERMS OF THE TIME-DEPENDENT DIELECTRIC SUSCEPTIBILITY}

The dielectric susceptibility $\chi$ links the induced polarization $\mathcal{P}$ to the field $\mathcal{E}$ via

$$
\mathcal{P}(t)=\int_{-\infty}^{t} d t^{\prime} \chi\left(t, t^{\prime}\right) \mathcal{E}\left(t^{\prime}\right) .
$$

The wave equation for light is then

$$
\nabla^{2} \mathcal{E}(t)-\frac{1}{c^{2}} \frac{\partial^{2} \mathcal{D}(t)}{\partial t^{2}}=0,
$$

where $\mathcal{D}(t)=\mathcal{E}(t)+4 \pi \mathcal{P}(t)$. The absorption coefficient $\alpha(\omega)$ is defined as the inverse of the length which light has to traverse in the medium at frequency $\omega$ in order for the intensity of the light to decrease by a factor of $1 / e$. In equilibrium, $\mathcal{D}(\omega)=[1+4 \pi \chi(\omega)] \mathcal{E}(\omega)=\epsilon(\omega) \mathcal{E}(\omega)$, and the absorption coefficient ${ }^{19}$ becomes

$$
\alpha(\omega)=4 \pi \omega \frac{\operatorname{Im} \chi(\omega)}{\operatorname{cn}(\omega)} .
$$

Here $n^{2}(\omega)=\frac{1}{2}[\operatorname{Re} \epsilon(\omega)+|\epsilon(\omega)|]$ is the refraction coefficient which usually depends only weakly on $\omega$. In nonequilibrium this analysis must be generalized, and we consider two special cases: (i) monochromatic continuous wave measurements, and (ii) white light short-pulse measurements.

\section{A. Continuous-wave measurements}

Consider a system out of equilibrium which is probed by a light field (which is assumed to be weak) of frequency $\omega_{l}$ :

$$
\mathcal{E}(r, t)=\mathcal{E}_{0} \exp \left[i\left(r k-\omega_{l} t\right)\right]
$$

The polarization can then be expressed as

$$
\mathcal{P}(t)=\mathcal{E}(r, t) \int_{-\infty}^{\infty} d t^{\prime} e^{i \omega_{l}\left(t-t^{\prime}\right)} \chi^{r}\left(t, t^{\prime}\right)
$$

This form is suggestive: it is advantageous to express $\chi^{r}$ in terms of the center-of-mass and difference coordinates, $\chi^{r}\left(t, t^{\prime}\right) \rightarrow \widetilde{\chi}^{r}\left[\frac{1}{2}\left(t+t^{\prime}\right), t-t^{\prime}\right]$. The characteristic time scale for the center-of-mass time is set by the "slow" frequency $\Omega$, while the difference time varies on the scale of the "fast" frequency $\omega_{l}$. We thus gradient expand $\widetilde{\chi}^{r}\left[\frac{1}{2}\left(t+t^{\prime}\right), t-t^{\prime}\right] \simeq \widetilde{\chi}^{r}\left(t, t-t^{\prime}\right)+\frac{1}{2}\left(t^{\prime}-t\right) \widetilde{\chi}^{r^{\prime}}\left(t, t-t^{\prime}\right)+\cdots$, where the prime indicates differentiation with respect to the slow temporal variable. Substitution in Eq. (33) then yields (we introduce a variable $\tau \equiv t-t^{\prime}$ )

$$
\begin{aligned}
\mathcal{P}(t) & =\mathcal{E}(r, t) \int d \tau e^{i \omega_{l} \tau}\left[\tilde{\chi}^{r}(t, \tau)+\left(-\frac{1}{2} \tau\right) \tilde{\chi}^{r^{\prime}}(t, \tau)+\cdots\right] \\
& =\mathcal{E}(r, t)\left[\tilde{\chi}^{r}\left(t, \omega_{l}\right)+\frac{\partial}{\partial \omega_{l}} \frac{\partial}{\partial t} \frac{i}{2} \tilde{\chi}^{r}\left(t, \omega_{l}\right)+\cdots\right] \\
& =\mathcal{E}(r, t) \exp \left[\frac{i}{2} \frac{\partial^{2}}{\partial t \partial \omega_{l}}\right] \tilde{\chi}^{r}\left(t, \omega_{l}\right)
\end{aligned}
$$

Equation (34) can now be used in the Maxwell equation; note, however, that upon Fourier transforming the dominant frequency comes from $\mathcal{E}(t)$, and we can keep $t$ in $\chi\left(t, \omega_{l}\right)$ fixed. The slow time variation will from this on be indicated by $T$. Proceeding as in deriving the static result (31), we identify the time dependent absorption coefficient

$$
\alpha_{T}(\omega)=4 \pi \omega \frac{\operatorname{Im} \tilde{\chi}^{r}(T, \omega)}{c n_{T}(\omega)}+\mathcal{O}(\Omega / \omega)
$$

If the driving force is periodic in $T$ (the harmonic time dependence due to a FEL laser is an important special case), then the average absorption coefficient is 


$$
\begin{aligned}
\bar{\alpha}(\omega) & =\frac{1}{T_{\text {period }}} \int_{\text {period }} d T \alpha_{T}(\omega) \\
& =\frac{1}{T_{\text {period }}} \int_{\text {period }} d T 4 \pi \omega \frac{\operatorname{Im} \widetilde{\chi}^{r}(T, \omega)}{c n_{T}(\omega)}
\end{aligned}
$$

to all orders in $\Omega / \omega$. We stress that here $\widetilde{\chi}^{r}(T, \omega)$ is Fourier transformed with respect to the difference variable $\tau$. Below we shall represent numerical examples for the generalized absorption coefficient.

\section{B. Short white light pulse measurements}

Consider now an instantaneous measurement performed on a nonequilibrium system: at some specific time $t=t_{m}$ the system is probed with a weak pulse whose duration is short compared to the characteristic dynamics of the system. We approximate the pulse with $\delta$ function in time:

$$
\mathcal{E}(r, t)=\mathcal{E}_{0} e^{i r k} \delta\left(t-t_{m}\right) .
$$

In principle, construction of such a pulse would take infinite energy due to its time dependence. The pulse is therefore hardly "weak." When we refer to the pulse as weak we assume that $\mathcal{E}_{0} \ll 1$; that is, the intensity of the light is small at all frequencies. Using Eq. (37) in the Maxwell equation yields the dispersion relation

$$
k^{2}=\frac{\omega^{2}}{c^{2}}\left[1+4 \pi \chi^{r}\left(\omega, t_{m}\right)\right]
$$

This dispersion relation looks quite similar to the one obtained in Sec. II. The difference is that here $\chi^{r}\left(\omega, t_{m}\right)$ is Fourier transformed with respect to $t^{\prime}$, not the difference variable $\tau$. In the present case we obtain the time-dependent absorption coefficient

$$
\alpha_{t}(\omega)=4 \pi \omega \frac{\operatorname{Im} \chi^{r}\left(\omega_{l}, t\right)}{c n_{t}(\omega)}
$$

For examples of experiments which probe systems in this manner, see, e.g., Refs. 10 and 11.

\section{Differential transmission spectrum}

Consider a sample of thickness $L$; then the ratio of the intensity transmitted through the sample with its initial intensity is $T(\omega)=\exp [-\bar{\alpha}(\omega) L]$, where $\bar{\alpha}(\omega)$ is the absorption coefficient of the sample. Experimental setups for measuring the change in absorption due to externally controlled perturbations commonly measure the differential transmission spectrum (DTS) defined by ${ }^{20}$

$$
\mathcal{D}(\omega)=\frac{T(\omega)-T_{0}(\omega)}{T_{0}(\omega)} .
$$

Here $T(\omega)$ is the transmission with the perturbation present and $T_{0}(\omega)$ is the transmission through the unperturbed sample. Below, we give examples of $\mathcal{D}(\omega)$ in nonequilibrium situations.

\section{SINGLE-PARTICLE GREEN FUNCTIONS AND SPECTRAL FUNCTIONS}

In this section we determine the single-particle Green functions and their associated spectral functions. We show that, under conditions specified below, that the convolutions of the spectral functions, encountered in Sec. II, result in effective single-band spectral functions.

\section{A. Single-particle Green functions}

Let $\vec{A}$ be the vector potential which defines the FIR field. Considering harmonic, translationally invariant external fields, we choose

$$
\vec{A}(t)=-\vec{E} \frac{\sin (\Omega t)}{\Omega},
$$

which represents the physical uniform electric field $\vec{E} \cos (\Omega t)$. The two-band single-particle Hamiltonian for a system subject to the external FIR field can generally be written in the form

$$
\begin{aligned}
H= & \sum_{\vec{k}}\left\{\epsilon_{c}\left[\vec{k}+\frac{e}{\hbar} \vec{A}\right] a_{\vec{k}}^{\dagger} a_{\vec{k}}+\epsilon_{v}\left[\vec{k}+\frac{e}{\hbar} \vec{A}\right] b_{\vec{k}}^{\dagger} b_{\vec{k}}\right\} \\
& +\sum_{\vec{k}, \vec{k}^{\prime}}\left\{F_{\vec{k}, \vec{k}^{\prime}}[\vec{A}(t)] a_{\vec{k}}^{\dagger} b_{\vec{k}^{\prime}}+\text { H.c. }\right\} .
\end{aligned}
$$

Here $F_{\vec{k}, \vec{k}},[\vec{A}(t)]$ describes the mixing of the bands due to Zener-like processes caused by the intense FIR field. We shall now argue that for realistic parameter values the mixing term can, in fact, be neglected. Let $\epsilon_{g}$ be the band gap $\left(\epsilon_{g} \simeq 1.4\right.$ for bulk GaAs). In order for the $\mathrm{THz}$ field to mix the bands, i.e., to yield a finite $F_{\vec{k}, \vec{k}^{\prime}}[\vec{A}(t)], 2 n$-photon processes have to occur with $n=\left[\epsilon_{g} / 2 \hbar \Omega\right]$. (Here $[x]$ indicates the integer part of $x$.) Note that only even-order photon processes are allowed due to parity. In Ref. 7, we showed that the $2 n$-photon process carries spectral weight $2 \pi J_{n}^{2}\left(\epsilon_{f} / 2 \hbar \Omega\right)$, where $\epsilon_{f}=e^{2} E^{2} / 4 m \Omega^{2}$ is the average classical kinetic energy obtained by an electron placed in the FIR field. Thus band mixing can be expected to be negligible if $J_{\left[\epsilon_{g} / \hbar \Omega\right]}^{2}\left(\epsilon_{f} / 2 \hbar \Omega\right) \ll 1$. Considering typical frequencies $(\hbar \Omega \simeq 2, \ldots, 20 \mathrm{meV})$ and strongest $\mathrm{THz}$ fields that are attainable in FEL facilities, $E \simeq 1 \mathrm{MV} / \mathrm{m}$, one finds that the argument of the Bessel function is of the order of unity. However, with these parameters $n$ is of the order of a few hundred, and since $J_{m}(x) \ll 1$ for $x \ll m,{ }^{28}$ we are indeed in a regime where band mixing is of no consequence and will henceforth be neglected.

The Dyson equation for the retarded and/or advanced free-particle Green function is

$$
\left(i \hbar \frac{\partial}{\partial t}-\epsilon_{\alpha}\left[\vec{k}+\frac{e}{\hbar} \vec{A}(t)\right]\right) g_{\alpha}^{r / a}\left(\vec{k}, t, t^{\prime}\right)=\delta\left(t-t^{\prime}\right),
$$

where $\alpha \in\{c, v\}$ is the band index. This equation is readily integrated with the solutions 


$$
\begin{aligned}
g_{\alpha}^{r / a}\left(\vec{k}, t, t^{\prime}\right)= & \mp \frac{i}{\hbar} \theta\left( \pm t \mp t^{\prime}\right) \\
& \times \exp \left\{-\frac{i}{\hbar} \int_{t^{\prime}}^{t} d s \epsilon_{\alpha}\left[\vec{k}+\frac{e}{\hbar} \vec{A}(s)\right]\right\},
\end{aligned}
$$

and the spectral function $A=i\left(g^{r}-g^{a}\right)$ becomes

$$
A_{\alpha}\left(\vec{k}, t, t^{\prime}\right)=\frac{1}{\hbar} \exp \left\{-\frac{i}{\hbar} \int_{t^{\prime}}^{t} d s \epsilon_{\alpha}\left[\vec{k}+\frac{e}{\hbar} \vec{A}(s)\right]\right\} .
$$

\section{B. Convolution of the spectral functions}

According to Sec. II, the susceptibility is obtained through the trace of a convolution of the spectral functions. We shall now show that within the present model the convolution of spectral functions results in an effective single-band spectral function.

In terms of the center of mass variables, $\tau=t-t^{\prime}$ and $T=\left(t+t^{\prime}\right) / 2$, we write the spectral functions as

$$
\begin{aligned}
A_{\alpha}(\vec{k}, T, \omega)= & \frac{1}{\hbar} \int_{-\infty}^{\infty} d \tau e^{i \omega \tau} \\
& \times \exp \left\{-\frac{i}{\hbar} \int_{T-\tau / 2}^{T+\tau / 2} d s \epsilon_{\alpha}\left[\vec{k}+\frac{e}{\hbar} \vec{A}(s)\right]\right\} .
\end{aligned}
$$

Then the convolution

$$
b\left(\vec{k}, T, \omega_{l}\right)=\hbar \int_{-\infty}^{\infty} \frac{d \omega}{2 \pi} A_{e}(\vec{k}, T, \omega) A_{v}\left(\vec{k}, T, \omega-\omega_{l}\right)
$$

of the two spectral functions becomes

$$
\begin{aligned}
b\left(\vec{k}, T, \omega_{l}\right)= & \frac{1}{\hbar} \int_{-\infty}^{\infty} d \tau e^{i \omega_{l} \tau} \exp \left\{-\frac{i}{\hbar} \int_{T-\tau / 2}^{T+\tau / 2} d s\right. \\
& \left.\times\left(\epsilon_{c}\left[\vec{k}+\frac{e}{\hbar} \vec{A}(s)\right]-\epsilon_{v}\left[\vec{k}+\frac{e}{\hbar} \vec{A}(s)\right]\right)\right\} .
\end{aligned}
$$

In the case of parabolic bands we have

$$
\epsilon_{c}[\vec{k}]=\frac{\hbar^{2} k^{2}}{2 m_{e}}, \quad \epsilon_{v}[\vec{k}]=-\frac{\hbar^{2} k^{2}}{2 m_{h}}-\epsilon_{g} .
$$

Here $m_{e}$ is the electron mass, $m_{h}$ is the positive hole mass, and $\epsilon_{g}$ is the band gap. We define a single effective band for the system,

$$
\epsilon_{\mathrm{eff}}[\vec{k}] \equiv \epsilon_{c}[\vec{k}]-\epsilon_{v}[\vec{k}]=\frac{\hbar^{2} k^{2}}{2 m_{\mathrm{eff}}}+\epsilon_{g}
$$

where $m_{\mathrm{eff}}=m_{e} m_{h} /\left(m_{e}+m_{h}\right)$ is the effective reduced mass. Thus the effective band is parabolic like the original bands, but with their reduced mass. It is therefore evident that the convolution, writing $A_{\text {eff }}\left(\vec{k}, T, \omega_{l}\right)=b\left(\vec{k}, T, \omega_{l}\right)$, is a spectral function for a parabolic band,

$$
\begin{aligned}
A_{\mathrm{eff}}(\vec{k}, T, \omega)= & \frac{1}{\hbar} \int_{-\infty}^{\infty} d \tau e^{i \omega \tau} \\
& \times \exp \left\{-\frac{i}{\hbar} \int_{T-\tau / 2}^{T+\tau / 2} d s \epsilon_{\mathrm{eff}}\left[\vec{k}+\frac{e}{\hbar} \vec{A}(s)\right]\right\}
\end{aligned}
$$

In the case of tight-binding minibands for a type-I superlattice (with period $a$ ), we write the bands as

$$
\begin{gathered}
\epsilon_{c}[\vec{k}]=\frac{1}{2} \lambda_{c} \cos \left(a k_{\|}\right)+\frac{\hbar^{2} k_{\perp}^{2}}{2 m_{e}}, \\
\epsilon_{v}[\vec{k}]=-\frac{1}{2} \lambda_{v} \cos \left(a k_{\|}\right)-\frac{\hbar^{2} k_{\perp}^{2}}{2 m_{h}}-\epsilon_{g},
\end{gathered}
$$

where $\lambda_{c}$ is the electron miniband width, $\lambda_{h}$ is the corresponding bandwidth for the holes, $k_{\|}$is the (crystal) momentum component parallel to the growth direction of the superlattice, and $k_{\perp}$ is the magnitude of the component perpendicular to the growth direction. The effective band thus becomes

$$
\epsilon_{\mathrm{eff}}[\vec{k}]=\frac{1}{2} \lambda_{\mathrm{eff}} \cos \left(a k_{\|}\right)+\frac{\hbar^{2} k_{\perp}^{2}}{2 m_{\mathrm{eff}}}+\epsilon_{g},
$$

where $\lambda_{\text {eff }}=\lambda_{c}+\lambda_{v}$ is the effective bandwidth, which again is of the same form as the original bands. This shows that also for superlattices the convolution (47) leads to an effective spectral function of the original form.

In terms of the effective spectral function the imaginary part of the susceptibility can be written as

$$
\operatorname{Im} \chi_{l m}^{r}\left(T, \omega_{l}\right)=\frac{d_{l} d_{m}}{2 \hbar} \sum_{\vec{k}} A_{\mathrm{eff}}\left(\vec{k}, T, \omega_{l}\right),
$$

where we assume that the dipole matrix elements are $k$ independent. In equilibrium the trace of the spectral function yields the density of states for the system. Analogously, the GDOS (Ref. 7) is defined as

$$
\rho\left(T, \omega_{l}\right)=\frac{1}{\pi} \sum_{\vec{k}} A_{\mathrm{eff}}\left(\vec{k}, T, \omega_{l}\right),
$$

allowing us to write the absorption coefficient as

$$
\alpha_{T}\left(\omega_{l}\right) \approx \frac{2 \pi^{2} \omega_{l}|d|^{2}}{c n \hbar} \rho\left(T, \omega_{l}\right)
$$

For the remainder of this work we shall investigate the properties of $\rho\left(T, \omega_{l}\right)$ for various systems. The concept of a timedependent density of states was discussed earlier in a specific context in Ref. 24. Here we have made the definition rigorous.

\section{Gauge invariance}

To conclude this section, we briefly comment on the gauge invariance. From the outset, we might have chosen to work within the gauge-invariant formulation which has been developed in the field of high-field transport. ${ }^{25,26,13}$ Consid- 
ering translationally invariant systems, correlation functions are made gauge invariant with the transformation

$$
\vec{k} \rightarrow \vec{k}+\frac{e}{t-t^{\prime}} \int_{t^{\prime}}^{t} \frac{d s}{\hbar} \vec{A}(s) .
$$

However, the absorption coefficient follows from a trace operation (56), which makes the transformation (58) redundant: a simple change of variables when performing the trace undoes Eq. (58), and proves that our formulation of the absorption is gauge invariant.

\section{PARABOLIC BANDS}

In this section we shall investigate the properties of the generalized density of states for systems which can be effectively described by Hamiltonians yielding parabolic bands, be it in one, two, or three dimensions. We write the effective single-band dispersion as

$$
\epsilon[\vec{k}]=\frac{\hbar^{2} k^{2}}{2 m_{\mathrm{eff}}}+\epsilon_{g} .
$$

For convenience we write $m=m_{\text {eff }}$, and set $\epsilon_{g}=0$ which shifts the energy axis such that the reference point is the band-gap energy. We calculate the generalized density of states from

$$
\rho^{n D}(T, \epsilon)=\int_{-\infty}^{\infty} d \tau e^{i \epsilon \tau / \hbar} \rho^{n D}(T, \tau),
$$

where

$$
\begin{aligned}
\rho^{n D}(T, \tau)= & \frac{1}{\hbar} \int \frac{d^{n} \vec{k}}{(2 \pi)^{n}} \\
& \times \exp \left\{-\frac{i}{\hbar} \int_{T-\tau / 2}^{T+\tau / 2} d s \in\left[\vec{k}+\frac{e}{\hbar} \vec{A}(s)\right]\right\} .
\end{aligned}
$$

With the vector potential (41), one obtains explicitly that

$$
\begin{aligned}
\rho^{n D}(T, \tau)= & \frac{1}{\hbar} \int \frac{d^{n} \vec{k}}{(2 \pi)^{n}} \exp \left\{-i\left[\left(\epsilon_{k}+\epsilon_{f}\right) \tau / \hbar\right.\right. \\
& +2 \frac{e \hbar \vec{k} \cdot \vec{E}}{m \Omega^{2}} \sin (\Omega T) \sin \left(\frac{\Omega \tau}{2}\right) \\
& \left.\left.-\frac{\omega_{f}}{\Omega} \cos (2 \Omega T) \sin (\Omega \tau)\right]\right\} .
\end{aligned}
$$

Here $\epsilon_{k}=\hbar^{2} k^{2} / 2 m$, and we have defined the fundamental energy scale, also recognized in Refs. 5 and 6,

$$
\epsilon_{f}=\hbar \omega_{f}=\frac{e^{2} E^{2}}{4 m \Omega^{2}} .
$$

The energy $\epsilon_{f}$ can be interpreted classically in the following way: consider a classical particle with charge $e$ and mass $m$ subjected to an electric field $\vec{E}(t)=\vec{E} \cos (\Omega t)$. From Newton's equation of motion one finds that the mean kinetic energy of such a particle equals $\epsilon_{f} \cdot{ }^{27}$
In order to perform the Fourier transform (60) we utilize the identity ${ }^{28}$

$$
\exp (i x \sin \theta)=\sum_{n} J_{n}(x) \exp (i n \theta),
$$

where $J_{n}(x)$ are Bessel functions; we shall henceforth write $\Sigma_{n} \equiv \Sigma_{n=-\infty}^{\infty}$ to simplify the notation. The generalized density of states becomes

$$
\begin{aligned}
\rho^{n D}(T, \epsilon)= & \sum_{l, j} \int \frac{d^{n} \vec{k}}{(2 \pi)^{n-1}} \delta\left[\epsilon-\epsilon_{k}-\epsilon_{f}+l \hbar \Omega\right] \\
& \times J_{2 j}\left(2 \frac{e \vec{k} \cdot \vec{E}}{m \Omega^{2}} \sin (\Omega T)\right) J_{l+j}\left(\frac{\omega_{f}}{\Omega} \cos (2 \Omega T)\right) .
\end{aligned}
$$

The dimensionality is entirely contained in the remaining momentum integration $\int d^{n} \vec{k} /(2 \pi)^{n-1}$. We note that Eq. (65) implies a shift of the absorption edge by $\epsilon_{f}$. The term $l \hbar \Omega$ in the Dirac $\delta$ function gives rise to photonic sidebands. Since $J_{2 l}(x)$ is an even function, the density of states is invariant under the transformation $\vec{E} \rightarrow-\vec{E}$, as expected. In the following subsections we shall consider the 1D, 2D, and 3D systems separately, and show how the density of states smoothly evolves from a low-field intensity regime into a high-field intensity regime, making the nonlinear effects of the $\mathrm{THz}$ field apparent.

\section{A. Generalized density of states, one dimension}

The density of states for a single-mode 1D system ("quantum wire") in the absence of external fields is

$$
\rho_{0}^{1 \mathrm{D}}(T, \epsilon)=\frac{1}{\pi}\left(\frac{2 m}{\hbar^{2}}\right)^{1 / 2} \epsilon^{-1 / 2} \theta(\epsilon) .
$$

In the presence of an external strong oscillating field, from (65) we obtain that the GDOS is

$$
\begin{aligned}
\rho^{1 \mathrm{D}}(T, \epsilon)= & \sum_{l, j} \int_{-\infty}^{\infty} d k \delta\left[\epsilon-\epsilon_{k}-\epsilon_{f}+l \hbar \Omega\right] \\
& \times J_{2 j}\left(\frac{\sqrt{32 \epsilon_{f} \epsilon_{k}}}{\hbar \Omega} \sin (\Omega T)\right) \\
& \times J_{l+j}\left(\frac{\epsilon_{f}}{\hbar \Omega} \cos (2 \Omega T)\right) \\
= & \sum_{l} r_{l}^{1 \mathrm{D}}\left(T, \epsilon-\epsilon_{f}+l \hbar \Omega\right) \rho_{0}^{1 \mathrm{D}}\left(\epsilon-\epsilon_{f}+l \hbar \Omega\right),
\end{aligned}
$$

where the sideband weights are

$$
\begin{aligned}
r_{l}^{1 \mathrm{D}}(T, \epsilon)= & \sum_{j} J_{2 j}\left(\frac{\sqrt{32 \epsilon_{f} \epsilon}}{\hbar \Omega} \sin (\Omega T)\right) \\
& \times J_{l+j}\left(\frac{\epsilon_{f}}{\hbar \Omega} \cos (2 \Omega T)\right) .
\end{aligned}
$$


We note that, in the limit $\epsilon_{f} \rightarrow 0$,

$$
r_{l}^{1 \mathrm{D}}(T, \epsilon) \rightarrow \delta_{l, 0},
$$

and $\rho^{1 \mathrm{D}}(T, \epsilon) \rightarrow \rho_{0}^{1 \mathrm{D}}(\epsilon)$, as expected. If $\Omega$ is in the $\mathrm{THz}$ regime, then most experiments would probe the timeaveraged absorption. The time average of the sideband weights is calculated from

$$
\begin{aligned}
\bar{r}_{l}^{1 \mathrm{D}}(\epsilon)= & \sum_{j} \int_{0}^{2 \pi} \frac{d s}{2 \pi} J_{2 j}\left(\frac{\sqrt{32 \epsilon_{f} \epsilon}}{\hbar \Omega} \sin (s)\right) \\
& \times J_{l+j}\left(\frac{\epsilon_{f}}{\hbar \Omega} \cos (2 s)\right),
\end{aligned}
$$

which for $l$ odd yields

$$
\begin{aligned}
\bar{r}_{l}^{1 \mathrm{D}}(\epsilon)= & \sum_{j} \int_{0}^{2 \pi} \frac{d s}{2 \pi} J_{4 j+2}\left(\frac{\sqrt{32 \epsilon_{f} \epsilon}}{\hbar \Omega} \sin (s)\right) \\
& \times J_{2 j+l+1}\left(\frac{\epsilon_{f}}{\hbar \Omega} \cos (2 s)\right),
\end{aligned}
$$

and, for $l$ even,

$$
\begin{aligned}
\bar{r}_{l}^{1 \mathrm{D}}(\epsilon)= & \sum_{j} \int_{0}^{2 \pi} \frac{d s}{2 \pi} J_{4 j}\left(\frac{\sqrt{32 \epsilon_{f} \epsilon}}{\hbar \Omega} \sin (s)\right) \\
& \times J_{2 j+l}\left(\frac{\epsilon_{f}}{\hbar \Omega} \cos (2 s)\right) .
\end{aligned}
$$

At the onset of sideband $l$, the sideband weight is

$$
\bar{r}_{l}^{1 \mathrm{D}}(0)=\left\{\begin{array}{cl}
0 & \text { if } l \text { odd } \\
J_{l / 2}^{2}\left(\epsilon_{f} / \hbar \Omega\right) & \text { if } l \text { even, }
\end{array}\right.
$$

where we used the identity ${ }^{28}$

$$
\int_{0}^{2 \pi} d \theta J_{2 l}(a \cos \theta)=2 \pi J_{l}^{2}(a)
$$

This shows that processes involving an odd number of photons of the $\mathrm{THz}$ field are strongly suppressed. In Fig. 1 we illustrate $\rho_{\mathrm{ave}}^{1 \mathrm{D}}(\epsilon)$ for a range of values of $\epsilon_{f} / \hbar \Omega$. In the figures we write $\epsilon_{e}=\hbar \Omega$. We observe all the signatures of the dynamical Franz-Keldysh effect (DFK): ${ }^{7}$ the Stark-like blueshift of the main absorption edge by $\epsilon_{f}$, the formation of sidebands at $\epsilon_{g}+\epsilon_{f} \pm N \hbar \Omega$, and finite absorption within the band gap.

\section{B. Generalized density of states, two dimensions}

Several authors considered fields perpendicular to the quantum well (cf. Ref. 29 and references therein); here we focus on the situation where the electric field is in the plane of the two-dimensional electron gas. In such a system with no external field, the density of states is constant,

$$
\rho_{0}^{2 \mathrm{D}}(\epsilon)=\frac{m}{\pi \hbar^{2}} \theta(\epsilon)
$$

With a harmonically oscillating field, we obtain, from Eq. (65),

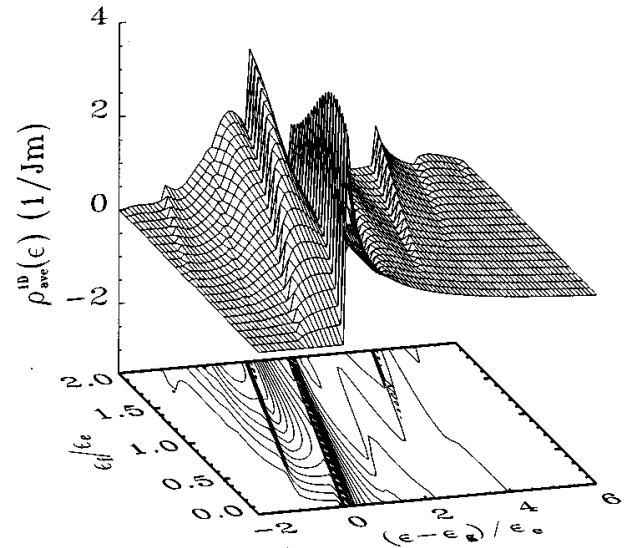

FIG. 1. Time-averaged generalized density of states for a 1D system shown for a range of FIR intensities, $\epsilon_{f} / \hbar \Omega \in[0,2]$. The band edge and the sidebands display a blueshift, which scales linearly with the intensity. Absorption extends below the band gap (dynamical Franz-Keldysh effect).

$$
\begin{aligned}
\rho^{2 \mathrm{D}}(T, \epsilon)= & \sum_{l, j} \int_{0}^{\infty} d k k \int_{0}^{2 \pi} \frac{d \theta}{2 \pi} \delta\left[\epsilon-\epsilon_{k}-\epsilon_{f}+l \hbar \Omega\right] \\
& \times J_{2 j}\left(\frac{\sqrt{32 \epsilon_{f} \epsilon_{k}}}{\hbar \Omega} \cos \theta \sin (\Omega T)\right) \\
& \times J_{l+j}\left(\frac{\epsilon_{f}}{\hbar \Omega} \cos (2 \Omega T)\right) .
\end{aligned}
$$

The integrals in Eq. (76) are again performed using Eq. (74), and writing the result in the sideband picture, we obtain ${ }^{30}$

$$
\rho^{2 \mathrm{D}}(T, \epsilon)=\sum_{l} r_{l}^{2 \mathrm{D}}\left(T, \epsilon-\epsilon_{f}+l \hbar \Omega\right) \rho_{0}^{2 \mathrm{D}}\left(\epsilon-\epsilon_{f}+l \hbar \Omega\right),
$$

where the sideband weights are

$$
r_{l}^{2 \mathrm{D}}(T, \epsilon)=\sum_{j} J_{j}^{2}\left(\frac{\sqrt{32 \epsilon_{f} \epsilon}}{\hbar \Omega} \sin (\Omega T)\right) J_{l+j}\left(\frac{\epsilon_{f}}{\hbar \Omega} \cos (2 \Omega T)\right) .
$$

Identical arguments as in the 1D case lead to

$$
\bar{r}_{l}^{2 \mathrm{D}}(0)=\left\{\begin{array}{cl}
0 & \text { if } l \text { odd } \\
J_{l / 2}^{2}\left(\epsilon_{f} / \hbar \Omega\right) & \text { if } l \text { even, }
\end{array}\right.
$$

i.e., the same result as in the $1 \mathrm{D}$ case.

As in the $1 \mathrm{D}$ case, we have numerically investigated the time averaged GDOS $\rho_{\text {ave }}^{2 \mathrm{D}}(\epsilon)=(\Omega / 2 \pi) \int_{0}^{2 \pi / \Omega} d T \rho^{2 \mathrm{D}}(T, \epsilon)$. In Fig. 2 we illustrate $\rho_{\text {ave }}^{2 \mathrm{D}}(\epsilon)$ for various values of $\epsilon_{f} / \hbar \Omega$. Again, as in the 1D case, we observe all the characteristics of the DFK. ${ }^{7}$ Finally, Fig. 3 shows the DTS signal.

\section{Generalized density of states, three dimensions}

Absorption in bulk semiconductors subject to $\mathrm{THz}$ radiation was considered a long time ago by Yacoby, ${ }^{5}$ and later by Rebane. ${ }^{6}$ These papers studied transition rates between bands by investigating approximate solutions to the corresponding time-dependent Schrödinger equation. Reference 5 con- 


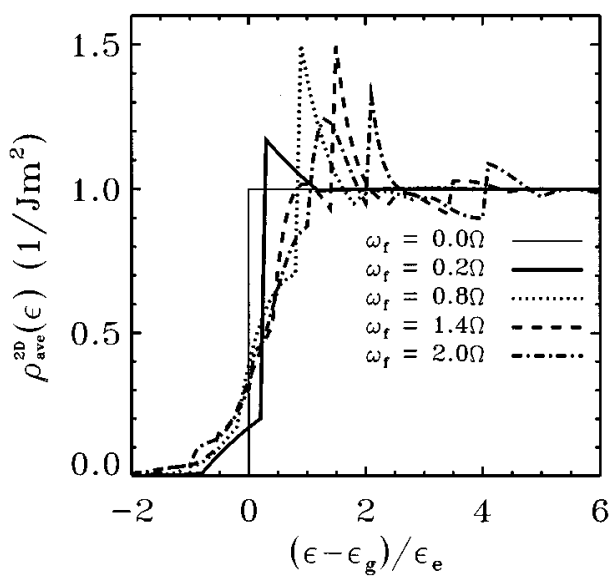

FIG. 2. The time-averaged GDOS for a 2D system for a range of FIR intensities, $\omega_{f} / \Omega=(0.0,0.2,0.8,1.4,2.0)$. At low intensities one observes a Stark-like blueshift of the band edge, as well as finite absorption within the band gap. With increasing intensity, sidebands emerge at $\epsilon=\epsilon_{g}+\epsilon_{f} \pm 2 \hbar \Omega$.

cluded that transitions occur in the gap, and noted reduced rates above the gap, in agreement with the present work, while Ref. 6 pointed out that the absorption egde would be shifted, likewise in agreement with our work. The 3D fieldfree density of states is

$$
\rho_{0}^{3 \mathrm{D}}(\epsilon)=\frac{1}{2 \pi^{2}}\left(\frac{2 m}{\hbar}\right)^{3 / 2} \theta(\epsilon) \epsilon^{1 / 2}
$$

With the external field the density of states becomes

$$
\rho^{3 \mathrm{D}}(T, \epsilon)=\sum_{l} r_{l}^{3 \mathrm{D}}\left(T, \epsilon-\epsilon_{f}+l \hbar \Omega\right) \rho_{0}^{3 \mathrm{D}}\left(\epsilon-\epsilon_{f}+l \hbar \Omega\right),
$$

with the sideband weights

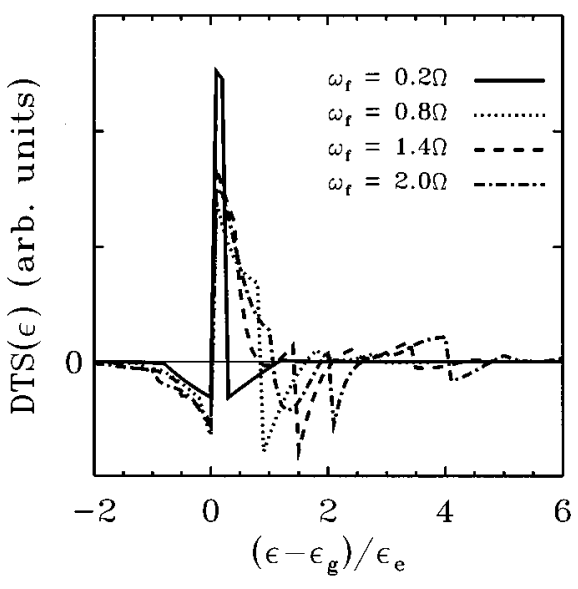

FIG. 3. The DTS signal for a 2D system for a range of intensities, $\omega_{f} / \Omega=(0.2,0.8,1.4,2.0)$.

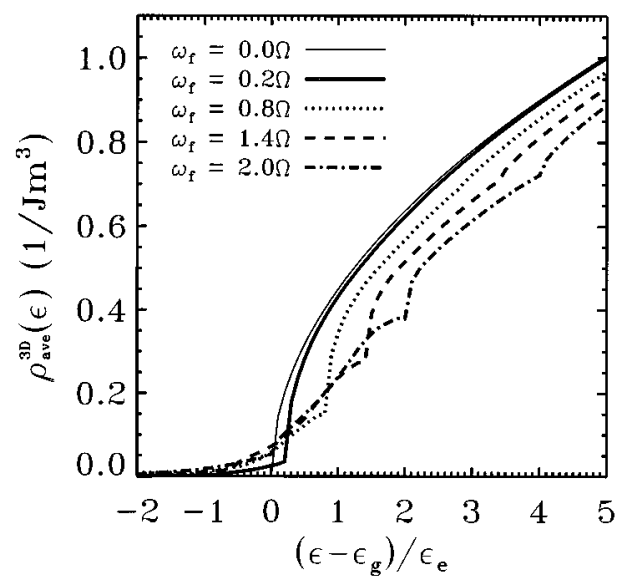

FIG. 4. The time-averaged generalized density of states for a 3D system for a range of FIR intensities, $\omega_{f} / \Omega=(0.0,0.2,0.8,1.4,2.0)$.

$$
\begin{aligned}
r_{l}^{3 \mathrm{D}}(T, \epsilon)= & \sum_{j} J_{l+j}\left(\frac{\epsilon_{f}}{\hbar \Omega} \cos (2 \Omega T)\right) \\
& \times \int_{0}^{1} d \eta J_{2 j}\left(\frac{\sqrt{32 \epsilon_{f} \epsilon}}{\hbar \Omega} \sin (\Omega T) \eta\right) .
\end{aligned}
$$

Again, we have

$$
\bar{r}_{l}^{3 \mathrm{D}}(0)=\left\{\begin{array}{cl}
0 & \text { if } l \text { odd } \\
J_{l / 2}^{2}\left(\epsilon_{f} / \hbar \Omega\right) & \text { if } l \text { even. }
\end{array}\right.
$$

In Fig. 4 we illustrate $\rho_{\text {ave }}^{3 \mathrm{D}}(\epsilon)$ for various values of $\epsilon_{f} / \hbar \Omega$; the DTS signal for the 3D case is shown in Fig. 5.

\section{Summary}

The main physical consequences of the $\mathrm{THz}$ field on the linear absorption spectrum for systems with parabolic dispersion can be summarized as follows. The dynamical modifications of the absorption spectrum (i) appear near the absorption edge, (ii) extend a few $\epsilon_{e}=\hbar \Omega$ around the edge, and (iii) are most pronounced when $\omega_{f} / \Omega$ is of order unity.

If $\Omega$ is in the THz regime, and fields like those attainable with free-electron lasers are considered, ${ }^{2,31}$ then $\omega_{f} / \Omega \approx 1$

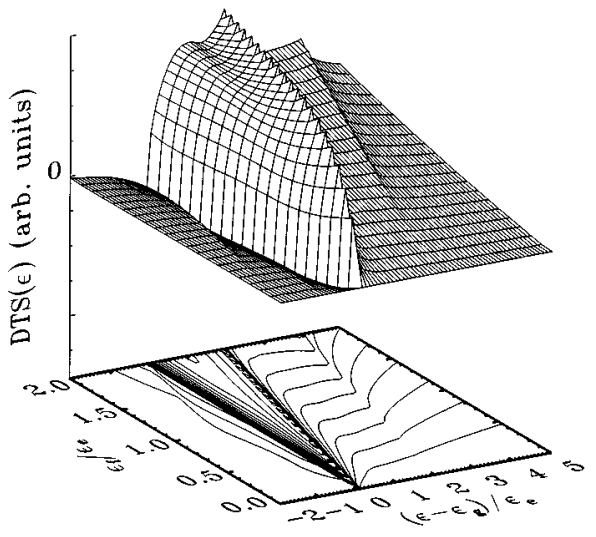

FIG. 5. DTS spectrum for a 3D system. 
and the fine structure extends over an area of several meV. Consequently, an experimental verification of these effects should be possible.

\section{SUPERLATTICES}

According to the semiclassical Bloch-Boltzmann theory of transport, a uniform electric field causes charge carriers in a periodic potential to execute a time-periodic motion with frequency $\omega_{B}=e a E / \hbar$, where $a$ is the lattice periodicity. Conditions for observing these Bloch oscillations are much more favorable in superlattices than in ordinary bulk materials, and recent years have witnessed an intense research effort culminating in the observation of Bloch oscillations. ${ }^{32}$ In ac fields a phenomenon called dynamical localization may occur: if the parameter $\gamma \equiv a e E_{\|} / \hbar \Omega$ equals a zero of $J_{0}$, the average velocity vanishes. ${ }^{33}$ In this section we investigate how dynamical localization ${ }^{4,33-36}$ manifests itself in the freeparticle absorption spectra. Recently, Meier et al. ${ }^{15}$ presented results of a detailed numerical solution of the semiconductor Bloch equations, including excitonic effects, and found that at dynamical localization the relative motion exciton wave function changes from a 3D character (i.e., localized in $k_{z}$ space) to a 2D structure (extended in $k_{z}$ space), and below we shall illustrate how the same phenomenon reflects itself in the present analytic study of free-particle properties.

\section{A. Generalized density of states}

The starting point for our analysis is the effective dispersion (54) introduced in Sec. IV B, which we reproduce here for the convenience of the reader:

$$
\epsilon_{\mathrm{eff}}[\vec{k}]=\frac{1}{2} \lambda_{\mathrm{eff}} \cos \left(a k_{\|}\right)+\frac{\hbar^{2} k_{\perp}^{2}}{2 m_{\mathrm{eff}}}+\epsilon_{g}
$$

Henceforth we put $\epsilon_{g}=0$, and drop the "eff'" subscript. We consider the effect of the $\mathrm{THz}$ field described by the vector potential $\vec{A}(t)=-\vec{E} \sin (\Omega t) / \Omega$, where $\vec{E}=\left(0,0, E_{\|}\right)$. In accordance with Sec. IV, we calculate the generalized density of states from

$$
\begin{aligned}
& \rho^{\mathrm{sl}}(T, \tau)= \frac{m}{2 \pi^{2} \hbar^{3}} \int_{0}^{\infty} d \epsilon_{\perp} \int_{0}^{2 \pi / a} d k_{\|} e^{-i \epsilon_{\perp} \tau / \hbar} \\
& \times \exp [-i I(T, \tau)], \\
& I(T, \tau)=\frac{\lambda}{2 \hbar} \int_{T-\tau / 2}^{T+\tau / 2} d s \cos \left[a k_{\|}+\gamma \sin (\Omega s)\right] .
\end{aligned}
$$

We evaluate the integral within the exponential using identity (64), with the result

$$
\begin{aligned}
I(T, \tau)= & \frac{\lambda}{2 \hbar \Omega}\left\{\cos \left(a k_{\|}\right)\left[\mathcal{C}(\Omega \tau)+J_{0}(\gamma) \frac{\lambda \tau}{2 \hbar}\right]\right. \\
& \left.+\sin \left(a k_{\|}\right) \mathcal{S}(\Omega \tau)\right\},
\end{aligned}
$$

where

$$
\begin{gathered}
\mathcal{C}(z)=2 \sum_{n=1}^{\infty} \frac{J_{2 n}(\gamma) \cos [2 n \Omega T]}{n} \sin [n z], \\
\mathcal{S}(z)=2 \sum_{n=1}^{\infty} \frac{J_{2 n-1}(\gamma) \sin [(2 n-1) \Omega T]}{n-1 / 2} \sin [(n-1 / 2) z] .
\end{gathered}
$$

We have suppressed the explicit dependence of $\Omega T$ and $\gamma$ in $\mathcal{C}(z)$ and $\mathcal{S}(z)$ for simplicity. Note that both of these functions are antisymmetric in $z$, i.e., $\mathcal{C}(-z)=-\mathcal{C}(z)$ and $\mathcal{S}(-z)=-\mathcal{S}(z)$. The identity ${ }^{28}$

$$
\int_{0}^{2 \pi} \frac{d \theta}{2 \pi} \exp \{i a \cos \theta+i b \sin \theta\}=J_{0}\left(\sqrt{a^{2}+b^{2}}\right)
$$

is the key to the next step in the evaluation of Eq. (85), and allows us to write

$$
\rho^{\mathrm{sl}}(T, \tau)=\frac{m}{2 \pi^{2} \hbar^{3} a} \int_{0}^{\infty} d \epsilon_{\perp} e^{-i \epsilon_{\perp} \tau / \hbar} \mathcal{K}(\Omega \tau)
$$

where we have defined the kernel

$$
\mathcal{K}(z)=J_{0}\left(\frac{\lambda}{2 \hbar \Omega} \sqrt{\left[\mathcal{C}(z)+J_{0}(\gamma) z\right]^{2}+\mathcal{S}^{2}(z)}\right) .
$$

Also here, we have suppressed the explicit dependence of $\Omega T$ and $\gamma$. In a distribution sense we can write

$$
\int_{0}^{\infty} d \epsilon_{\perp} e^{-i \epsilon_{\perp} \tau / \hbar}=\frac{-i \hbar}{\tau-i 0^{+}}
$$

where $0^{+}$indicates a positive infinitesimal. This expression allows us to compute the Fourier transform of Eq. (91),

$$
\rho^{\mathrm{sl}}(T, \epsilon)=\frac{m}{2 \pi^{2} \hbar^{2} a}\left(\int_{-\infty}^{\infty} d \tau \frac{\sin (\epsilon \tau / \hbar)}{\tau} \mathcal{K}(\Omega \tau)+\pi\right)
$$

In what follows we shall examine several properties of this result.

\section{B. Field-free limit}

In the limit of vanishing field strength we have

$$
\lim _{\gamma \rightarrow 0} \mathcal{K}(z)=J_{0}\left(\frac{\lambda z}{2 \hbar \Omega}\right)
$$

Using the identity ${ }^{28}$

$$
\int_{-\infty}^{\infty} \frac{d x}{x} \sin (\beta x) J_{0}(x)= \begin{cases}\pi & {[\beta>1]} \\ 2 \arcsin \beta & {\left[\beta^{2}<1\right]} \\ -\pi & {[\beta<-1],}\end{cases}
$$

we obtain the density of states for a tight-binding superlattice, 


$$
\rho^{\mathrm{sl}}(\epsilon)=\frac{m}{\pi \hbar^{2} a} \begin{cases}1 & {[\epsilon>\lambda / 2]} \\ \frac{1}{\pi} \arcsin (2 \epsilon / \lambda)+1 / 2 & {[|\epsilon| \leqslant \lambda / 2]} \\ 0 & {[\epsilon<-\lambda / 2],}\end{cases}
$$

which is familiar.

\section{Static limit}

In the limit $\Omega \rightarrow 0$ one obtains

$$
\lim _{\Omega \rightarrow 0} \mathcal{K}(\Omega \tau)=J_{0}\left(\frac{\lambda}{2 \hbar \omega_{B}} \sin \left(\omega_{B} \tau\right)\right) .
$$

Recalling the identities ${ }^{28}$

$$
J_{0}(z \sin \alpha)=\sum_{j} J_{j}^{2}(z / 2) \cos (2 k \alpha)
$$

and

$$
\int_{-\infty}^{\infty} d x \cos (\alpha x) \sin (\beta x) / x=\pi[\theta(\alpha+\beta)-\theta(\alpha-\beta)],
$$

we obtain the density of states

$$
\rho^{\mathrm{sl}}(\epsilon)=\frac{m}{\pi \hbar^{2} a} \sum_{j} J_{j}^{2}\left(\frac{\lambda}{4 \hbar \omega_{B}}\right) \theta\left(\epsilon+2 j \hbar \omega_{B}\right)
$$

This result coincides with the one obtained in Refs. 37 and 38 , which studied both theoretically and experimentally the effects of strong static fields on the absorption in superlattice structures. They concluded that the steplike behavior of (101) is due to localization in the growth direction (WannierStark localization).

\section{Dynamic localization}

As seen in Sec. IV C, the signature of localization in the growth direction in a superlattice is a steplike behavior of the density of states. This is intuitively clear since the density of states for a 2D system [Eq. (75)] is constant. We therefore expect the density of states to be composed of a step function for each well the states extend into, with weight relative to the occupation in that particular well. We shall now show that if $J_{0}(\gamma)=0$, i.e., the conditions for dynamical localization are met, then GDOS indeed is of this kind. The argument runs as follows. If $J_{0}(\gamma)=0$ then kernel (92) is periodic in $z$ with period $2 \pi$. Furthermore, the kernel is an even function: $\mathcal{K}(z)=\mathcal{K}(-z)$. We can therefore formally write

$$
\mathcal{K}(\Omega \tau)=\sum_{j} \mathcal{K}_{j} \cos (k \Omega \tau),
$$

which is of the same functional form as in the static limit [Eq. (98)]. Consequently, we may conclude that the generalized density of states must be of the form

$$
\rho_{\mathrm{dyn} . \operatorname{loc}}^{\mathrm{sl}}(\epsilon)=\frac{m}{\pi \hbar^{2} a} \sum_{j} \mathcal{K}_{j} \theta(\epsilon+j \hbar \Omega)
$$

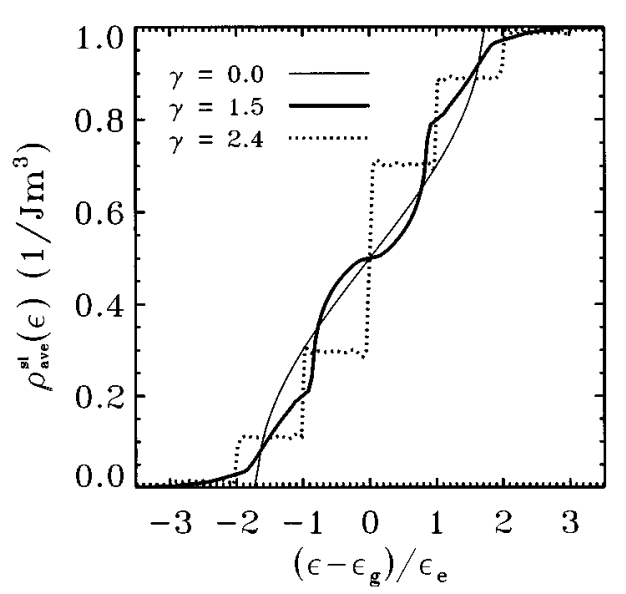

FIG. 6. The time averaged GDOS for a superlattice with effective bandwidth $\lambda=3.4 \hbar \Omega$. The proximity of dynamical localization occurring at $\gamma=2.4048 \ldots$ reflects itself in the stepwise structure of the dashed curve.

i.e., it is a superposition of step functions. The weights $K_{j}$, however, must be evaluated numerically, and examples are given in Sec. VIE. It is important to note that the "step length" in the ac case is determined by the frequency of the $\mathrm{THz}$ field, in contrast to the static case, where it is determined by the field strength. The field strength enters the density of states only through the weight factors $K_{j}$.

Result (102) suggests that it should be possible to probe dynamic localization by photoabsorption: when the appropriate conditions are approached, the absorption coefficient should change qualitatively from a generic smooth behavior to a sharply defined steplike structure. The number of distinct steps appearing in the spectrum is determined by the ratio $\lambda / \hbar \Omega$, which is also a measure of the number of wells the localized states span. This is fully consistent with the results of Ref. 15, whose authors considered a miniband of width $\lambda=21 \mathrm{meV}$ and a FIR frequency $\hbar \Omega=20 \mathrm{meV}$, which essentially allows just one step, and hence a maximum binding energy of the corresponding exciton which would be

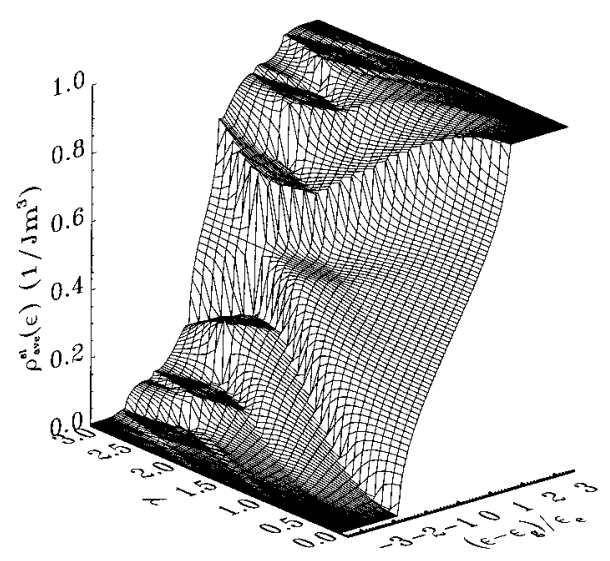

FIG. 7. The time-averaged GDOS for a superlattice with $\lambda=3.4 \hbar \Omega$ as a function of FIR intensity. At low $\gamma$, sidebands are observed, which merge at $\gamma=2.4048 \ldots$, corresponding to dynamical localization. 
mostly confined to a single well. Interestingly, Eq. (102) suggests an interpretation of the feature at $1534 \mathrm{meV}$, which Meier et al. found "unexpected:" in our picture it is the photon replica of the main exciton peak; since this occurs at $\simeq 1552 \mathrm{meV}$ and the photon energy is $20 \mathrm{meV}$, the agreement is very good indeed.

\section{E. Numerical results}

We again focus our numerical study to the time-averaged generalized density of states

$$
\rho_{\mathrm{ave}}^{\mathrm{sl}}(\epsilon)=(\Omega / 2 \pi) \int_{0}^{2 \pi / \Omega} d T \rho^{\mathrm{sl}} \times(T, \epsilon) .
$$

In Figs. 6 and 7 we show the absorption spectra for a superlattice with an effective bandwidth $\lambda=3.4 \hbar \Omega$. The numerical results confirm the expectations of Sec. VI D: when $\gamma=a e E_{\|} / \hbar \Omega$ approaches the first zero of $J_{0}$, which occurs at the argument value of $2.4048 \ldots$, the gradually evolving replicas of the zero-field density of states converge into plateaus of finite width. The exactness of the plateaus can be judged from Fig. 7: at $\gamma=2.4048 \ldots$, the line joining the the steps appears near vertical. Finally, in Fig. 8 we show the DTS spectra at dynamical localization (DL) and non-DL conditions. There are two characteristic differences: (i) Outside the zero-field miniband, DL leads to a steplike structure in contrast to the smooth behavior found otherwise; and (ii) inside the miniband the DL spectrum distinguishes itself by its sharp jagged structure.

\section{CONCLUSIONS}

We have presented a theoretical formulation of linear photoabsorption for samples under strongly nonequilibrium conditions. Typical nonequilibrium agents would be $\mathrm{THz}$ radiation from free-electron lasers, or ultrashort pulse measurements of transient effects. In the present work noninteracting carriers are considered, but the formulation allows an extension to Coulomb interactions, which will be addressed in a

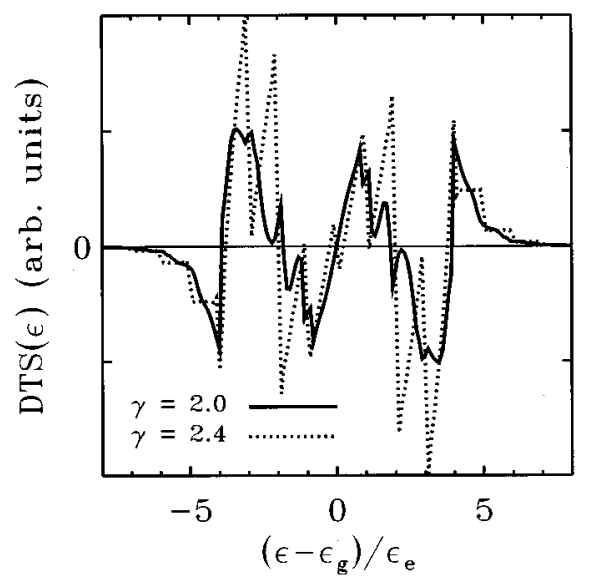

FIG. 8. The differential transmission signal for a superlattice structure with an effective minibandwidth $\lambda=8 \hbar \Omega$. Outside the zero-field miniband, a steplike behavior is seen, while inside the miniband DTS for dynamical localization develops a jagged shape in contrast to the smooth behavior for the extended state.

subsequent paper. Two central concepts emerge from our analysis: a generalization of the density of states into timedependent conditions [the GDOS defined in Eq. (56)], and photonic sidebands (i.e., photon replicas), which form a convenient framework for discussing the various features of the absorption spectra.

\section{ACKNOWLEDGMENTS}

We wish to acknowledge useful discussions with Ben $\mathrm{Hu}$, Andreas Wacker, and Björn Birnir. Furthermore, we wish to thank Fausto Rossi and Junichiro Kono for valuable comments of an earlier version of the manuscript and Kent Nordstrom for sharing the details of his unpublished data (Ref. 17). This work was partially supported by a LACOR Grant No. 4157U0015-3A from Los Alamos National Laboratory.
${ }^{1}$ J. Cerne et al., Phys. Rev. B 51, 5253 (1995).

${ }^{2}$ P. S. S. Guimarães et al., Phys. Rev. Lett. 70, 3792 (1993); B. J. Keay et al., ibid. 75, 4098 (1995).

${ }^{3}$ B. J. Keay et al., Phys. Rev. Lett. 75, 4102 (1995).

${ }^{4}$ M. Holthaus and D. W. Hone, Phys. Rev. B 49, 16605 (1994).

${ }^{5}$ Y. Yacoby, Phys. Rev. 169, 610 (1968).

${ }^{6}$ Yu. T. Rebane, Fiz. Tverd. Tela (Leningrad) 27, 1364 (1985)

[Sov. Phys. Solid State 27, 824 (1985)].

${ }^{7}$ A.-P. Jauho and K. Johnsen, Phys. Rev. Lett. 76, 4576 (1996).

${ }^{8}$ J. Kono et al., Phys. Rev. Lett. 79, 1758 (1997); J. Kono et al., in Proceedings of the $23^{\text {rd }}$ International Conference on the Physics of Semiconductors, edited by M. Scheffler and R. Zimmerman (World Scientific, Singapore, 1996), p. 1911.

${ }^{9}$ B. Birnir et al., Phys. Rev. B 47, 6795 (1995).

${ }^{10}$ H. Heesel et al., Phys. Rev. B 47, 16000 (1993).

${ }^{11}$ F. X. Camecasse et al., Phys. Rev. Lett. 77, 5429 (1996).

${ }^{12}$ For a recent theoretical paper modeling the experiments of Ref. 3 see, e.g., A. Wacker et al., in cond-mat/9612035 (unpublished), where other relevant work is also cited.

${ }^{13}$ H. Haug and A.-P. Jauho, Quantum Kinetics in Transport and Optics of Semiconductors (Springer, Berlin, 1996).

${ }^{14}$ W. Pötz, Phys. Rev. B 54, 5647 (1996).

${ }^{15}$ T. Meier, F. Rossi, P. Thomas, and S. W. Koch, Phys. Rev. Lett. 75, 2558 (1995).

${ }^{16}$ These rules are summarized, e.g., in Quantum Kinetics in Transport and Optics of Semiconductors (Ref. 13), Table 4.1.

${ }^{17}$ H. Haug and S. Schmitt-Rink, Prog. Quantum Electron. 9, 3 (1984).

${ }^{18}$ K. Nordstrom et al., Phys. Status Solidi B 204, 52 (1997).

${ }^{19}$ The reader should note that the symbol $\tau$ is used in a dual meaning: it can indicate a complex-time variable on the Keldysh contour, or the time-difference variable. Confusion can be avoided by noting that in the case of complex-time variables the Green functions have a superscript $c$, while in the real-time case they have $r, a$, or $<$.

${ }^{20} \mathrm{H}$. Haug and S. W. Koch, Quantum Theory of the Optical and 
Electronic Properties of Semiconductors (World Scientific, Singapore, 1993).

${ }^{21}$ T. Kuhn and F. Rossi, Phys. Rev. Lett. 69, 977 (1992); 72, 152 (1994); S. Haas et al., Phys. Rev. B 53, 12855 (1996).

${ }^{22}$ See, e.g., Quantum Kinetics in Transport and Optics of Semiconductors (Ref. 13), Chap. 11.

${ }^{23}$ S. Schmitt-Rink, D. S. Chemla, and D. A. Miller, Adv. Phys. 38, 89 (1989).

${ }^{24}$ P. K. Tien and J. P. Gordon, Phys. Rev. 129, 647 (1963).

${ }^{25}$ J. H. Davies and J. W. Wilkins, Phys. Rev. B 38, 1667 (1988).

${ }^{26}$ R. Bertoncini and A.-P. Jauho, Phys. Rev. B 44, 3655 (1991).

${ }^{27}$ The same concept is also known in atomic and plasma physics as the ponderomotive energy, for a set of reviews see Atoms in Intense Laser Fields, edited by M. Gavrila, Advances in Atomic, Molecular, and Optical Physics Suppl. 1 (Academic, New York, 1992).

${ }^{28}$ I. S. Gradshteyn and I. M. Ryzhik, Table of Integrals, Series and Products, 5th ed. (Academic, New York, 1994).
${ }^{29}$ M. Wagner, Phys. Rev. Lett. 76, 4010 (1996); J. Inarrea and G. Platero, Europhys. Lett. 34, 43 (1996).

${ }^{30}$ It is interesting to note that by comparing the GDOS in the 2D and 3D cases with Eqs. (15)-(18) in Ref. 7, one may obtain exact expansions for the integrals in terms of Bessel functions.

${ }^{31}$ K. Unterrainer et al., Phys. Rev. Lett. 76, 2973 (1996).

${ }^{32}$ C. Waschke et al., Phys. Rev. Lett. 70, 3319 (1993).

${ }^{33}$ A. A. Ignatov and Y. A. Romanov, Phys. Status Solidi B 73, 327 (1976); D. H. Dunlap and V. M. Kenkre, Phys. Rev. B 34, 3625 (1986).

${ }^{34}$ X.-G. Zhao, J. Phys.: Condens. Matter 6, 2751 (1994).

${ }^{35}$ M. Holthaus, G. W. Ristow, and D. W. Hone, Europhys. Lett. 32, 241 (1995).

${ }^{36}$ J. Rotvig, A.-P. Jauho, and H. Smith, Phys. Rev. Lett. 74, 1831 (1995); Phys. Rev. B 54, 17691 (1996).

${ }^{37}$ J. Bleuse, G. Bastard, and P. Voisin, Phys. Rev. Lett. 60, 220 (1988).

${ }^{38}$ P. Voisin et al., Phys. Rev. Lett. 61, 1639 (1988). 